Check for updates

Cite this: RSC Adv., 2017, 7, 23924

\title{
FRET efficiency and antenna effect in multi-color DNA origami-based light harvesting systems $\uparrow$
}

\begin{abstract}
L. Olejko ${ }^{\mathrm{abc}}$ and I. Bald (D) *ab
Artificial light harvesting complexes find applications in artificial photosynthesis, photovoltaics and light harvesting chemical sensors. They are used to enhance the absorption of light of a reaction center which is often represented by a single acceptor. Here, we present different light harvesting systems on DNA origami structures and analyze systematically the light harvesting efficiency. By changing the number and arrangement of different fluorophores (FAM as donor, Cy3 as transmitter and Cy5 as acceptor molecules) the light harvesting efficiency is optimized to create a broadband absorption and to improve the antenna effect 1 (including two energy transfer steps) from 0.02 to 1.58 , and the antenna effect 2 (including a single energy transfer step) from 0.04 to 8.7, i.e. the fluorescence emission of the acceptor is significantly higher when the light-harvesting antenna is excited at lower wavelength compared to direct excitation of the acceptor. The channeling of photo energy to the acceptor proceeds by Förster Resonance Energy Transfer (FRET) and we carefully analyze also the FRET efficiency of the different light harvesting systems. Accordingly, the antenna effect can be tuned by modifying the stoichiometry of donor, transmitter and acceptor dyes, whereas the FRET efficiency is mainly governed by the spectroscopic properties of dyes and their distances.
\end{abstract}

Received 20th February 2017

Accepted 25th April 2017

DOI: $10.1039 / \mathrm{c} 7 \mathrm{ra02114c}$

rsc.li/rsc-advances of chromophores can be combined and multi-color photonic networks can be assembled. Here, the principle of photonic wires is exploited. Photonic wires are optical waveguides, in which the light energy is controlled on the nanoscale by transferring it linearly along multiple fluorophores from one end to the other end based on FRET. ${ }^{\mathbf{6} 15-19}$ Several FRET cascades can be combined in a star-like arrangement to transfer the light energy from the outside (donor molecules) to the center of the photonic network (acceptor dye). By combing these two photonic assemblies (light harvesting complexes with FRET cascades) larger end-to-end distances can be achieved and, above all, a broader range of wavelengths from the electromagnetic spectrum can be absorbed to a higher extent leading to a better light harvesting efficiency for several wavelengths. Additionally, the light harvesting efficiency of such systems can be improved on the one hand by optimizing the parameters determining the FRET process, i.e. through a larger spectral overlap of donor (D) and acceptor (A) molecules and shorter D-A distances. On the other hand, the number and spatial arrangement of $\mathrm{D}$ and $\mathrm{A}$ molecules can be varied. The distance and number of $\mathrm{D}$ and A dyes can be accurately controlled by using DNA scaffolds. This has been demonstrated e.g. with different star-like DNA nanostructures, ${ }^{\mathbf{1 6}}$ however, the relative flexibility of the arms of these DNA structures is rather high. DNA origami nanostructures ${ }^{20}$ offer a higher control over the arrangement of a larger number of dye molecules without changing the geometry of the DNA substrate. These DNA nanostructures which are assembled by molecular self-
Str. 24-25, 14476 Potsdam, Germany. E-mail: olejko@uni-potsdam.de

${ }^{b}$ BAM Federal Institute for Materials Research and Testing, Richard-Willstätter Str. 11, 12489 Berlin, Germany.E-mail: bald@uni-potsdam.de

${ }^{c}$ School of Analytical Sciences Adlershof, Humboldt-Universität zu Berlin, Unter den Linden 6, 10099 Berlin, Germany

$\uparrow$ Electronic supplementary information (ESI) available. See DOI: 10.1039/c7ra02114c 
assembly using a circular virus DNA strand called "scaffold strand" (here: M13mp18, 7249 nucleobases (nb)) and many short single DNA strands called "staple strands" (208 different staple strands, $32 \mathrm{nb})^{20}$ are a versatile tool to arrange and analyze different molecules with a high local control and a variety of applications. ${ }^{21,22}$ Since every staple strand can be addressed and modified individually and separately, different moieties can be arranged with a high local control since the exact position of each staple strand in the DNA origami structure is known. DNA origami structures have been used to create highly sensitive SERS substrates by attaching gold nanoparticle dimers, ${ }^{23-25}$ to analyze DNA strand breaks induced by low energy electrons ${ }^{26,27}$ and UV photons ${ }^{28}$ and to arrange different fluorophores ${ }^{29,29,30}$ at precise distances to create nanoscale photonic devices which can be used for example as photonic wires, ${ }^{15,18}$ to resolve conformational changes of biomolecules, ${ }^{31-34}$ as logic gates $^{35,36}$ and artificial light harvesting complexes. ${ }^{8,10,18}$ The light harvesting efficiency is in this context typically expressed as an antenna effect (AE), i.e. the intensity of A emission when $\mathrm{D}$ is excited compared to the A intensity when it is directly excited..$^{6,8,10,16,37,38}$ An antenna is therefore formed when $\mathrm{AE}$ is higher than 1 , i.e. A is getting brighter by collecting light at lower wavelength compared to the direct excitation wavelength. Very recently, it has been demonstrated by using a DNA origami template that $\mathrm{AE}$ can be increased to higher values than 1 by increasing the number of D molecules (up to six). ${ }^{8}$ However, to screen a broader range of possible excitation wavelengths another energy transfer step, i.e. a third dye needs to be introduced. Here, we design and analyze different light harvesting systems on DNA origami structures using three different fluorophores. We focus in particular on the question how the stoichiometry of $\mathrm{D}$ and transmitter $(\mathrm{T})$ molecules affect both the $\mathrm{AE}$ and the FRET efficiency in the two-step energy transfer with up to eight D molecules. In general, the FRET efficiency characterizes the energy transfer with respect to the donor fluorescence, whereas the antenna effect is determined from the acceptor properties. Due to the presence of the third dye we also study the effect of the relative positioning of $\mathrm{D}, \mathrm{T}$ and $\mathrm{A}$, i.e. the difference between a linear, cross-like arrangement and a snowflake arrangement (in which the donors are rotated by $90^{\circ}$ ). For all arrangements we carefully determine antenna effects and the three-color FRET efficiencies.

\section{Results and discussion}

\section{Design of the artificial light harvesting system}

In this study, we have designed light harvesting antennas on triangularly shaped DNA origami structures (AFM image shown in the ESI Fig. S1 $\dagger$ ). For this, one acceptor molecule (Cyanine5, Cy5) is placed in the center of the light harvesting system. This acceptor molecule is surrounded by a maximum number of four transmitter molecules (Cyanine3, Cy3) (see also Fig. 1). The transmitter molecules are either placed on a neighboring DNA double helix (Fig. 1: paths I and III, T1 and T3) resulting in a nominal T-A distance of $c a .4 \mathrm{~nm}$ (the fluorophores are on opposite sides of the DNA origami structure, detailed fluorophore orientation is shown in the ESI in Fig. S2 †) or they are

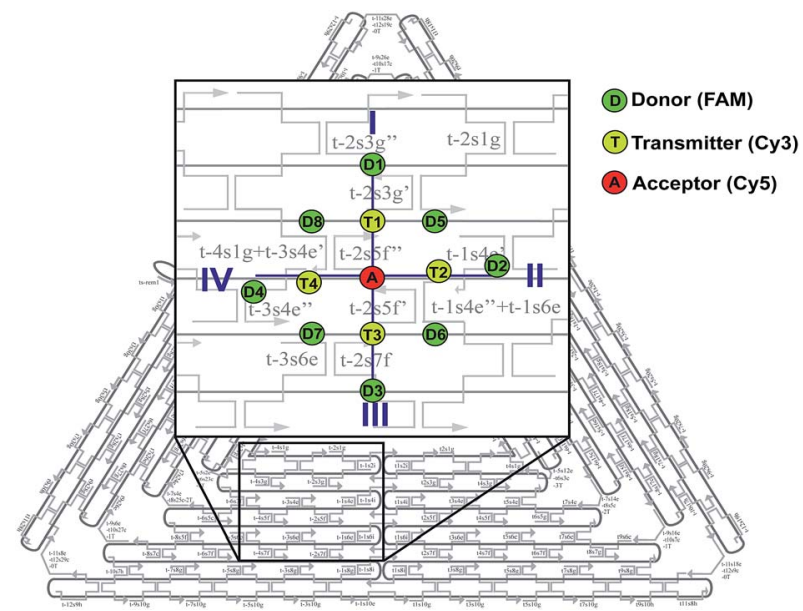

Fig. 1 Illustration of the artificial, FRET-based light harvesting complex on DNA origami structures with three different chromophores (donor: fluorescein (FAM), transmitter: Cyanine3 (Cy3), acceptor: Cyanine5 (Cy5)). The acceptor dye Cy5 (red) is placed in the middle of the light harvesting complex. A maximum number of four transmitter dyes (yellow) is placed around Cy5 (two on neighboring DNA double helices (gap size $=0.5 \mathrm{~nm}$; Cy5-Cy3-distance: ca. $4 \mathrm{~nm}$; path: I, III; T1 and T3) and two along the same DNA double helix ( $9 \mathrm{nb}$ away from Cy5 on opposite sides; Cy5-Cy3-distance: ca. 3 nm, path: II, IV; T2 and T4)). One donor molecule (green) is placed next to each transmitter molecule. Again, either on a neighboring DNA double helix (Cy3-FAMdistance: ca. $4 \mathrm{~nm}$; path: I, III; D1 and D3) or along the same DNA double helix (Cy3-FAM-distance: ca. $3 \mathrm{~nm}$; path: II, IV; D2 and D4). Additionally, FAM is placed between two Cy3 molecules having similar distances to each Cy3 ( $R=3.3 \mathrm{~nm}$, D5-D8). The light harvesting efficiency is influenced by the amount of donor and transmitter dyes around the central acceptor dye.

placed along the same DNA double helix with a distance of $9 \mathrm{nb}$ between Cy5 and Cy3 (9 nb $\approx 3 \mathrm{~nm}$ ) (Fig. 1: paths II and IV, T2 and T4). Next to each transmitter molecule a donor molecule (fluorescein, FAM) is placed. Again, the fluorophores are either placed on a neighboring DNA double helix (Fig. 1: paths I and III, D1 and D3) or along the same DNA double helix (distance = $9 \mathrm{nb}$, Fig. 1: paths II and IV, D2 and D4). In addition to these four FAM molecules, FAM can be placed at equal distance to two Cy3 molecules (such that FAM, two Cy3 molecules and Cy5 form a square, e.g. A-T1-D5-T2). Here, every FAM molecule (Fig. 1, D5D8) has a similar distance to the two neighboring Cy3 molecules $(\approx 3.3 \mathrm{~nm})$. In such a fluorophore arrangement high FRET efficiencies are expected because the intermolecular distances are shorter than the corresponding Förster distances $\left(R_{0}(\mathrm{Cy} 3 /\right.$ Cy5) $=5.2 \mathrm{~nm} ; R_{0}(\mathrm{FAM} / \mathrm{Cy} 3)=6.7 \mathrm{~nm}$; see Table $\mathrm{S} 1$ in the ESI $\dagger$ ). In this study, artificial light harvesting systems with a maximum number of eight FAM molecules can be created. This nanoscale photonic array can be excited at $450 \mathrm{~nm}$ (direct excitation of FAM) and due to an energy transfer from FAM over Cy3 to Cy5 it emits at $665 \mathrm{~nm}$. The emission intensity and therefore the light harvesting efficiency of the system can be tuned by changing the combination of used fluorophores and donor/transmitter/acceptor ratios.

The light harvesting efficiency can be expressed in different ways. On the one hand, the light harvesting efficiency can be 
determined by comparing the absorption spectrum with the fluorescence excitation spectrum recorded at the emission wavelength of the central acceptor dye. The decrease in intensity of the donor excitation peak compared to the donor's absorption maximum (normalizing of the absorption and excitation spectra is necessary) represents the transfer efficiency. ${ }^{\mathbf{4 0 , 4 1}}$ However, in the present experiments the concentrations of the dyes are in the range of $n M$, which prevents the detection of absorption spectra. On the other hand, the AE is understood as the relative emission intensity of the acceptor when the donor or the acceptor is excited, respectively. Hence, to characterize the light harvesting efficiency the $\mathrm{AE}$ is used as defined below. The AE resembles the acceptor emission generated by neighboring donors (sensitized emission) relative to the acceptor emission upon direct excitation..$^{\mathbf{6 , 8 , 1 0 , 1 6 , 3 7 - 3 9}}$ The overall antenna effect (AE1, FAM-Cy3-Cy5-FRET) is calculated using the following eqn (1).

$$
\mathrm{AE} 1=\frac{I_{\mathrm{A}(450 \mathrm{~nm})}}{I_{\mathrm{A}(600 \mathrm{~nm})}}
$$

Thus, it is the ratio of the acceptor emission intensity due to FRET $I_{\mathrm{A}(450 \mathrm{~nm})}$ (number in brackets denotes the excitation wavelength) and the acceptor emission intensity upon direct excitation at $600 \mathrm{~nm} I_{\mathrm{A}(600 \mathrm{~nm})}$ of the same sample (both emission intensities are corrected for equal photon fluxes). ${ }^{\mathbf{8 , 1 0 , 1 6 , 4 2}}$ For the three-color FRET cascade an antenna effect for the second FRET step (AE2, Cy3-Cy5-FRET) can be determined based on the direct excitation of the transmitter dye (here: Cy3; excitation wavelength $\left.=500 \mathrm{~nm} ; I_{\mathrm{A}(500 \mathrm{~nm})}\right)$.

$$
\mathrm{AE} 2=\frac{\left.I_{\mathrm{A}(500} \mathrm{nm}\right)}{\left.I_{\mathrm{A}(600} \mathrm{nm}\right)}
$$

It has to be noted that the exact value of the antenna effect depends strongly on the wavelength chosen for direct acceptor excitation, which has been intensively studied by Hemmig et al. ${ }^{\mathbf{8}}$ Therefore, when comparing AE values one has to carefully use the same wavelengths and measurement settings for every sample. In the present study, an excitation wavelength is chosen that allows to record the whole emission spectrum of Cy5 (thus, the excitation wavelength is not necessarily the absorption maximum).

The FRET efficiency $E$ is calculated based on the donor's fluorescence decay time using eqn (3)..$^{\mathbf{4 3 4}}$

$$
E=1-\frac{\bar{\tau}_{\mathrm{DA}}}{\tau_{\mathrm{D}}}
$$

Here, $\bar{\tau}_{\mathrm{DA}}$ is the donor's fluorescence decay time when FRET takes place (amplitude averaged fluorescence decay time) and $\tau_{\mathrm{D}}$ is the donor's fluorescence decay time with the acceptor being absent. To analyze the overall FRET efficiency and to take the contributions of all species (different donor-transmitteracceptor distances, unquenched FAM etc.) into account the amplitude averaged decay has been used to calculate the FRET efficiency. The donor's decay curves in absence and presence of the acceptor/transmitter molecules have been recorded using time-correlated single photon counting.

\section{Antenna effect}

We have analyzed different fluorophore assemblies and determined the antenna effects AE1 and AE2 and the FRET efficiencies for each system (AE2 is shown in the ESI, Fig. S3†). The determination of all these quantities yields a complete description of the energy transfer pathways in such light harvesting antennas.

Firstly, we have characterized the two-color FRET system using FAM as the donor and Cy5 as the acceptor molecule. Since the spectral overlap between these two organic dyes (see ESI, Fig. $\mathrm{S} 4 \mathrm{~A} \dagger$ ) is rather small and the distance between FAM and Cy5 ( $\approx 5-6 \mathrm{~nm}$ ) is relatively long the light harvesting efficiency is expected to be rather small for this system. Nevertheless, the light harvesting efficiency of such a two-color FRET pair can be improved by increasing the number of donor molecules ((Cy5) $(\text { Cy3 })_{0}(\mathrm{FAM})_{1-4}$, Fig. 2A, fluorophore which is increased is written in bold letters) which has also been shown in other studies. ${ }^{\mathbf{8 1 2}, 13}$ The emission intensity of Cy5 (665 nm) increases with additional donor molecules as shown in the normalized emission spectra in Fig. 2A (normalized to Cy5 emission intensity when Cy5 is directly excited; normalized emission spectra represent the overall functionality of the light harvesting system because emission of Cy5 in not correctly formed light harvesting systems is also taken into account). Please note that the peak at $533 \mathrm{~nm}$ in the emission spectrum of (Cy5) $(\mathrm{Cy} 3)_{0}(\mathrm{FAM})_{0}$ is the water Raman peak, which is also present in other emission spectra. Since the number of FAM molecules increases and FRET is rather weak, the emission intensity of FAM (520 nm) also increases stepwise (Fig. 2A). As depicted in Fig. 2D (black curve) the overall light harvesting efficiency increases with the addition of donor molecules. Please note that the AE1 values are mean values for each path and path combination (1× FAM: I, II, III, IV; $2 \times$ FAM: I + II, I + III, I + IV, II + III, II + IV, III + IV; $3 \times$ FAM: I + II + III, I + II + IV, I + III + IV, II + III + IV; see ESI Fig. S5A $\uparrow$ for further information). The error bars in Fig. 2D are the standard deviations of different paths and path combination and for systems with only one combination possibility $\left((\mathrm{Cy} 5)_{1}(\mathrm{Cy} 3)_{0}(\mathrm{FAM})_{0},(\mathrm{Cy} 5)_{1}(\mathrm{Cy} 3)_{0}(\mathrm{FAM})_{4}\right.$ and $(\mathrm{Cy} 5)_{1}$ $\left.(\mathrm{Cy} 3)_{4}(\mathrm{FAM})_{4}\right)$ they are the standard deviation of three independent measurements. The overall antenna effect is rather small due to weak FRET between FAM and Cy5 as explained above (AE1 range: $0.02 \pm 0.01$ to $0.32 \pm 0.01$ ). Since the antenna effect cannot be tuned arbitrarily using this two-color FRET system a transmitter molecule (Cy3) is introduced (Fig. 1). The normalized emission spectra for the stepwise addition of Cy3 to the two-color FRET system with the highest antenna effect $\left((\mathrm{Cy} 5)_{1}(\mathrm{Cy} 3)_{0}(\mathrm{FAM})_{4}\right)$ is shown in Fig. 2B. Here, the emission intensity of Cy3 $(565 \mathrm{~nm})$ increases with an increasing number of Cy3 molecules. This is on the one hand due to the raising amount of Cy3 molecules (see also ESI, Fig S6†), and on the other hand the FRET efficiency from FAM to Cy3 increases, which is also visible by a drop in the FAM emission intensity at $520 \mathrm{~nm}$ (Fig. 2B). Please note that the emission peak of FAM for 


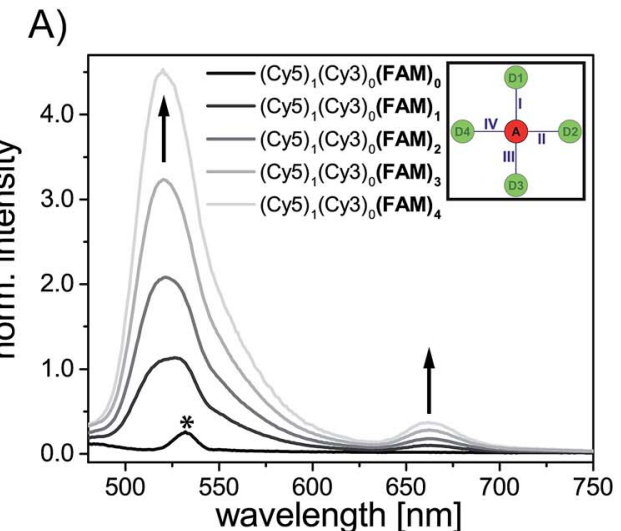

C)

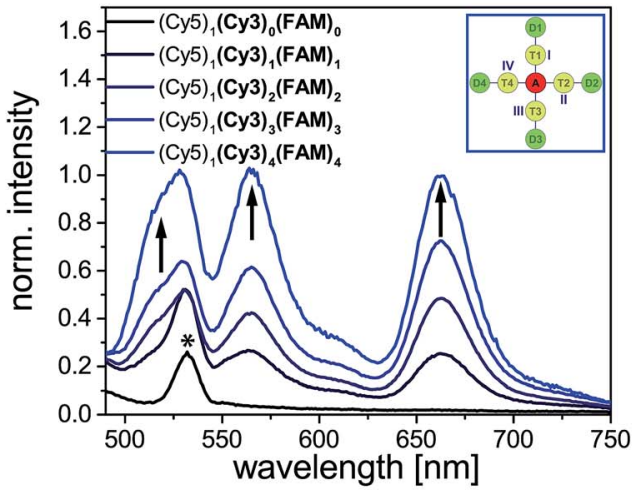

B)

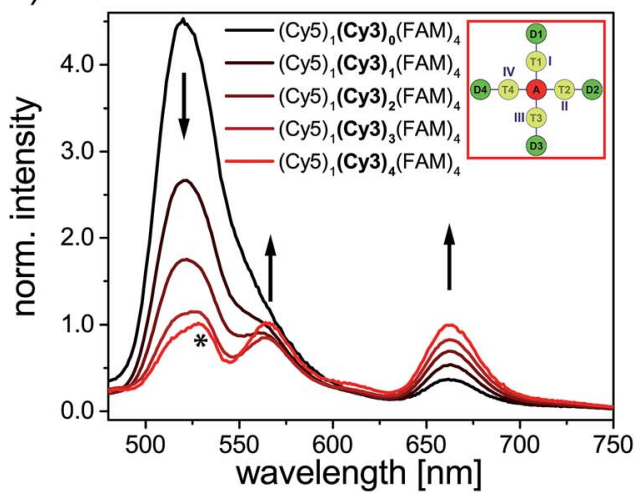

D)

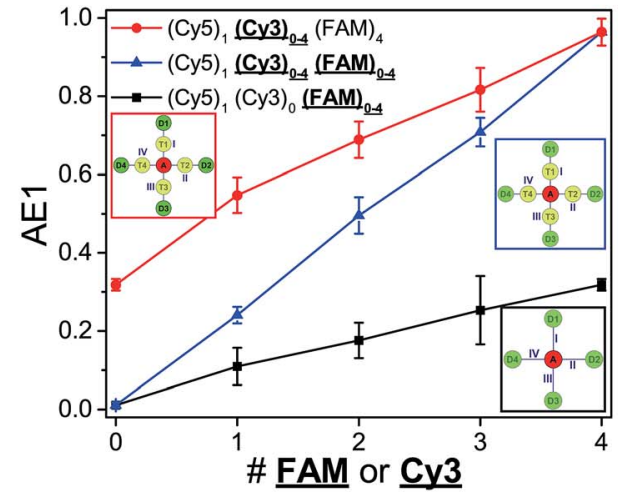

Fig. 2 Normalized emission spectra ( $\lambda_{\mathrm{ex}}=450 \mathrm{~nm}$, water Raman peak labelled with a star) and calculated antenna effect (AE1) for different light harvesting systems (mean values of different paths and path combinations). (A) Normalized emission spectra of the two-color FRET system $\left((\mathrm{Cy} 5)_{1}(\mathrm{Cy} 3)_{0}(\mathrm{FAM})_{0-4}\right)$ with increasing FAM molecules. The emission intensities of both FAM (520 nm) and Cy5 (665 nm) increase with increasing number of FAM molecules. (B) Light harvesting system with a fixed number of Cy5 and FAM molecules and increasing number of Cy3 molecules $\left((\mathrm{Cy} 5)_{1}(\mathrm{Cy} 3)_{0-4}(\mathrm{FAM})_{4}\right)$. Emission intensities of $\mathrm{Cy} 3$ and $\mathrm{Cy} 5$ increase and at the same time FAM emission intensity decreases due to higher FRET

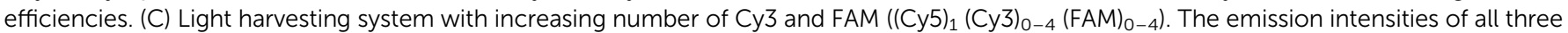
dyes increase due to a higher amount of molecules (FAM, Cy3) and therefore more light energy is transferred to Cy5. (D) The antenna effect increases for all systems with an increasing number of FAM and/or Cy3 molecules indicated in bold letters in the respective system. The overall $\mathrm{AE} 1$ of $(\mathrm{Cy} 5)_{1}(\mathrm{Cy} 3)_{0}(\mathrm{FAM})_{0-4}$ (black) is rather small due to weak FRET between FAM and Cy5. The increase in AE1 for (Cy5) ${ }_{1}(\mathrm{Cy} 3)_{0-4}(\mathrm{FAM})_{0-4}$ (blue) is steeper because two effects lead to an increase in AE1 in this light harvesting system. Namely, direct FAM-Cy5-FRET (stays the same for $\left.(C y 5)_{1}(C y 3)_{0-4}(F A M)_{4}(r e d)\right)$ increases and three-color FRET increases. Please note that the number of FAM or Cy3 on the $x$-axis refers to the number of fluorophores which are increased as indicated by highlighted letters in the caption.

the system $(\mathrm{Cy} 5)_{1}(\mathrm{Cy} 3)_{4}(\mathrm{FAM})_{4}$ is altered due to the underlying water Raman peak at $533 \mathrm{~nm}$. The overall emission intensity of Cy5 increases as well due to better energy transfer from FAM over Cy3 to Cy5. Furthermore, the antenna effect increases linearly with an increasing number of $\mathrm{Cy} 3$ molecules as shown in Fig. 2D (red curve). Here, the antenna effect can be further tuned from $0.32 \pm 0.01$ to $0.96 \pm 0.03$. This is very close to a value of 1 , which means that the light harvesting system is good enough to provide the same emission of A when excited at $450 \mathrm{~nm}$ as for direct A excitation at $600 \mathrm{~nm}$, although two energy transfer steps are required. Due to larger spectral overlaps between FAM/Cy3 and Cy3/Cy5 (see ESI, Fig. S4A-D, Table S1 $\dagger$ ) and shorter intermolecular distances the energy transfer becomes more efficient and therefore the sensitized emission of Cy5 is intensified. Again, the values of the antenna effect represent mean values of different paths and pathway combinations as mentioned above (see also ESI, Fig. S5C $\dagger$ ). The AE2 values for these systems also increase linearly and are shown in the ESI (Fig. S3A and C†). The AE2 values range from $3.0 \pm 0.2$ to $5.7 \pm 0.4$ showing that the brightness of the light harvesting complex also depends on the overall excitation wavelength $\left(\lambda_{\mathrm{ex}}\right.$ $\left.=450 \mathrm{~nm}(\mathrm{AE} 1) v s . \lambda_{\mathrm{ex}}=500 \mathrm{~nm}(\mathrm{AE} 2)\right)$. Interestingly, the AE2 values are significantly higher than 1 meaning that the light harvesting system can be very efficiently excited at $500 \mathrm{~nm}$. This can be attributed to the fact, that at $500 \mathrm{~nm}$ both FAM and Cy3 are excited. Thus, more light energy is transferred to the final acceptor Cy5. This shows that by using a three-color FRET cascade for a light-harvesting complex a broader range of wavelengths can be used to efficiently generate acceptor emission.

We have also analyzed the light harvesting system with an increasing number of both Cy3 and FAM. The amount of both fluorophores has been increased to the same extent ((Cy5) $(\mathrm{Cy} 3)_{1}(\mathrm{FAM})_{1},(\mathrm{Cy} 5)_{1}(\mathrm{Cy} 3)_{2}(\mathrm{FAM})_{2},(\mathrm{Cy} 5)_{1}(\mathrm{Cy} 3)_{3}(\mathrm{FAM})_{3}$ and $\left.(\mathrm{Cy} 5)_{1}(\mathrm{Cy} 3)_{4}(\mathrm{FAM})_{4}\right)$. The normalized emission spectra for these light harvesting systems are shown in Fig. 2C. Here, the 
fluorescence emission intensities of all fluorophores (Cy5, Cy3, FAM) increase with an increasing number of Cy3 and FAM (Fig. 2C). Due to a higher number of donor and transmitter molecules more light energy is transferred to the final acceptor. Therefore, the emission intensity of Cy5 at $665 \mathrm{~nm}$ increases gradually (Fig. 2C). Please note that the peak at $533 \mathrm{~nm}$ for $(\mathrm{Cy} 5)_{1}(\mathrm{Cy} 3)_{0}(\mathrm{FAM})_{0}$ is the water Raman peak as mentioned above and the emission peak of FAM for all light harvesting systems $\left((\mathrm{Cy} 5)_{1}(\mathrm{Cy} 3)_{1}(\mathrm{FAM})_{1},(\mathrm{Cy} 5)_{1}(\mathrm{Cy} 3)_{2} \mathrm{FAM}\right)_{2},(\mathrm{Cy} 5)_{1}(\mathrm{Cy} 3)_{3}$ $(\mathrm{FAM})_{3}$ and $\left.(\mathrm{Cy} 5)_{1}(\mathrm{Cy} 3)_{4}(\mathrm{FAM})_{4}\right)$ is altered due to the water Raman peak. The light harvesting efficiency increases with higher numbers of $\mathrm{Cy} 3$ and FAM as shown in Fig. 2D (blue curve). The antenna effect increases again linearly but compared to $(\mathrm{Cy} 5)_{1}(\mathrm{Cy} 3)_{0-4}(\mathrm{FAM})_{4}$ the slope is steeper. This is attributed to the fact that for $(\mathrm{Cy} 5)_{1}(\mathrm{Cy} 3)_{0-4}(\mathrm{FAM})_{4}$ the direct energy transfer from FAM to Cy5 stays the same (number of FAM molecules is constant) and in $(\mathrm{Cy} 5)_{1}(\mathrm{Cy} 3)_{0-4}(\mathrm{FAM})_{0-4}$ the direct FAM-to-Cy5 FRET increases (increasing number of FAM molecules), which leads to a stronger increase of the light harvesting efficiency. Furthermore, the antenna effect is always smaller for $(\mathrm{Cy} 5)_{1}(\mathrm{Cy} 3)_{0-4}(\mathrm{FAM})_{0-4}$ when compared to (Cy5) $(\mathrm{Cy} 3)_{0-4}(\mathrm{FAM})_{4}$. This is again because of the direct FAM-Cy5 FRET which is always higher in (Cy5) ${ }_{1}(\mathrm{Cy} 3)_{0-4}(\mathrm{FAM})_{4}$ (higher number of FAM molecules; 4 vs. $0-3)$. The AE1 for all paths and path combinations and the antenna effect 2 for $(\mathrm{Cy} 5)_{1}(\mathrm{Cy} 3)_{1-4}$ $(\mathrm{FAM})_{1-4}$ are shown in the ESI (Fig S5E and S3B and $\mathrm{C}, \dagger$ respectively). The AE2 values increase also linearly and range from $1.5 \pm 0.1$ in $(\mathrm{Cy} 5)_{1}(\mathrm{Cy} 3)_{1}(\mathrm{FAM})_{1}$ to $5.7 \pm 0.3$ in $(\mathrm{Cy} 5)_{1}$ $(\mathrm{Cy} 3)_{4}(\mathrm{FAM})_{4}$. The overall behavior when comparing the AE2 values of $(\mathrm{Cy} 5)_{1}(\mathrm{Cy} 3)_{1-4}(\mathrm{FAM})_{1-4}$ and $(\mathrm{Cy} 5)_{1}(\mathrm{Cy} 3)_{1-4}(\mathrm{FAM})_{4}$ with each other is the same as for AE1 (slope is steeper and values are smaller for $\left.(\mathrm{Cy} 5)_{1}(\mathrm{Cy} 3)_{1-4}(\mathrm{FAM})_{1-4}\right)$. The reason for this has been discussed above.

\section{FRET efficiency}

After analyzing the steady-state fluorescence data we have also carried out time-resolved fluorescence measurements to further characterize the different light harvesting systems in terms of FRET efficiency (Fig. 3). The FAM fluorescence decay curves have been analyzed using a multi-exponential fit as explained in the ESI $\dagger$ (two-exponential for $(\mathrm{Cy} 5)_{1}(\mathrm{Cy} 3)_{0}(\mathrm{FAM})_{1-4}$ and threeexponential for $(\mathrm{Cy} 5)_{1}(\mathrm{Cy} 3)_{0-4}(\mathrm{FAM})_{4},(\mathrm{Cy} 5)_{1}(\mathrm{Cy} 3)_{1-4}(\mathrm{FAM})_{1-4}$ and $\left.(\mathrm{Cy} 5)_{1}(\mathrm{Cy} 3)_{4}(\mathrm{FAM})_{8}\right)$. For all systems the last decay time component was set to the free FAM fluorescence decay time $\left(\bar{\tau}_{\mathrm{D}}\right.$ $=4.1-4.6 \mathrm{~ns}$ ) (the FAM fluorescence decay time depends on the position of FAM on the DNA origami structure, an overview of FAM fluorescence decay times of the different positions and position combinations is shown in the ESI (Table S $2 \dagger$ )).

The fluorescence decay curves for the two-color FRET system $(\mathrm{Cy} 5)_{1}(\mathrm{Cy} 3)_{0}(\mathrm{FAM})_{1-4}$ are depicted in Fig. 3A and the corresponding FRET efficiencies are plotted in Fig. 3D (black curve). The amplitude averaged decay time decreases from $4.3 \mathrm{~ns}$ in $(\mathrm{Cy} 5)_{0}(\mathrm{Cy} 3)_{0}(\mathrm{FAM})_{4}$ to $3.4 \mathrm{~ns}$ in $(\mathrm{Cy} 5)_{1}(\mathrm{Cy} 3)_{0}(\mathrm{FAM})_{1}$ due to direct FAM to Cy5 FRET. By increasing the number of FAM molecules stepwise the amplitude averaged decay time increases slightly $\left((\mathrm{Cy} 5)_{1}(\mathrm{Cy} 3)_{0}(\mathrm{FAM})_{1}: \bar{\tau}_{\mathrm{DA}}=(3.4 \pm 0.5) \mathrm{ns}\right.$;
$(\mathrm{Cy} 5)_{1}(\mathrm{Cy} 3)_{0}(\mathrm{FAM})_{2}: \bar{\tau}_{\mathrm{DA}}=(3.5 \pm 0.2) \mathrm{ns} ;(\mathrm{Cy} 5)_{1}(\mathrm{Cy} 3)_{0}(\mathrm{FAM})_{3}$ : $\left.\bar{\tau}_{\mathrm{DA}}=(3.5 \pm 0.1) \mathrm{ns} ;(\mathrm{Cy} 5)_{1}(\mathrm{Cy} 3)_{0}(\mathrm{FAM})_{4}: \bar{\tau}_{\mathrm{DA}}=(3.6 \pm 0.1) \mathrm{ns}\right)$. Thus, the FRET efficiency which has been calculated based on the FAM fluorescence decay times (amplitude averaged decay times) decreases slightly (Fig. 3D, black curve). The FRET efficiencies plotted in Fig. 3D are mean values of all paths and path combinations (see ESI, Fig. S5B $\dagger$ ). Again, the error bars are the standard deviations of different paths and path combinations or the standard deviation of three separate measurements for $(\mathrm{Cy} 5)_{1}(\mathrm{Cy} 3)_{0}(\mathrm{FAM})_{4}$ and $(\mathrm{Cy} 5)_{1}(\mathrm{Cy} 3)_{4}(\mathrm{FAM})_{4}$ (Fig. 3D). The standard deviations of different paths and path combinations represent the structural heterogeneity (variations in donor/ transmitter/acceptor distances and fluorophore orientations due to coupling to DNA) in the light harvesting design (see also ESI, Fig. S5 $\dagger$ ). The decrease in FRET efficiency can be explained by the manner of data analysis as follows: since we have determined the amplitude averaged fluorescence decay time, the decay time of unquenched FAM is taken into account. As we only increase the number of FAM molecules and FRET between FAM and Cy5 is rather weak, the amplitude of the decay time component for the free FAM increases (see also ESI, Fig. S7A $\dagger$ ). This leads to an increase in the amplitude averaged decay time and therefore a decrease in FRET efficiency. The first decay time component belonging to the quenched FAM due to direct FAMCy5 FRET stays on average nearly the same for all cases (see also ESI, Fig. S7B $\dagger)$. The overall FRET efficiency is rather small $(E \approx$ 0.2 ) in this system because of the small spectral overlap between FAM and Cy5 (see ESI, Fig. S4A $\dagger$ ) and the relatively long intermolecular distance $(\approx 5-6 \mathrm{~nm})$ as mentioned above. In general, this indicates that when FRET is of low efficiency the average FRET efficiency is further decreased upon increasing the number of D molecules. As we have seen above and will further discuss below, for high FRET efficiencies the average FRET efficiency is further increased upon increasing the number of $\mathrm{D}$ molecules.

Next, we consider the FRET efficiency for the three-color cascade (including Cy3). The FAM fluorescence decay curves for an increasing number of Cy3 molecules and a fixed number of Cy5 $(1 \times$ Cy5 $)$ and FAM $(4 \times$ FAM $)$ molecules are shown in Fig. 3B. The FAM amplitude averaged fluorescence decay time decreases with an increasing amount of Cy3 $\left((\mathrm{Cy} 5)_{1}(\mathrm{Cy} 3)_{0}\right.$ $(\mathrm{FAM})_{4}: \bar{\tau}_{\mathrm{DA}}=(3.6 \pm 0.1) \mathrm{ns} ;(\mathrm{Cy} 5)_{1}(\mathrm{Cy} 3)_{1}(\mathrm{FAM})_{4}: \bar{\tau}_{\mathrm{DA}}=(2.9 \pm$ $0.2) \mathrm{ns} ;(\mathrm{Cy} 5)_{1}(\mathrm{Cy} 3)_{2}(\mathrm{FAM})_{4}: \bar{\tau}_{\mathrm{DA}}=(2.4 \pm 0.4) \mathrm{ns} ;(\mathrm{Cy} 5)_{1}(\mathrm{Cy} 3)_{3}$ $(\mathrm{FAM})_{4}: \bar{\tau}_{\mathrm{DA}}=(1.8 \pm 0.5) \mathrm{ns} ;(\mathrm{Cy} 5)_{1}(\mathrm{Cy} 3)_{4}(\mathrm{FAM})_{4}: \bar{\tau}_{\mathrm{DA}}=(1.3 \pm$ $0.2) \mathrm{ns}$ ). The corresponding FRET efficiencies plotted against the number of Cy3 molecules are shown in Fig. 3D (red curve). Please note that the FRET efficiencies are again mean values of different paths and path combinations (see ESI, Fig. S5D†). The FRET efficiency rises with an increasing amount of Cy3. For $(\mathrm{Cy} 5)_{1}(\mathrm{Cy} 3)_{1}(\mathrm{FAM})_{4}$ the FRET efficiency is still rather small $(E=$ $0.32 \pm 0.02$ ) because one Cy3 molecule is not enough to transfer the light energy of four FAM molecules. The more Cy3 molecules are present the better the light energy is transferred ( $E$ increases). This is also in accordance with the antenna effect (Fig. 1D, red curve) discussed above. A stepwise addition of Cy3 molecules results in a linear increase of the FRET efficiency. 
A)

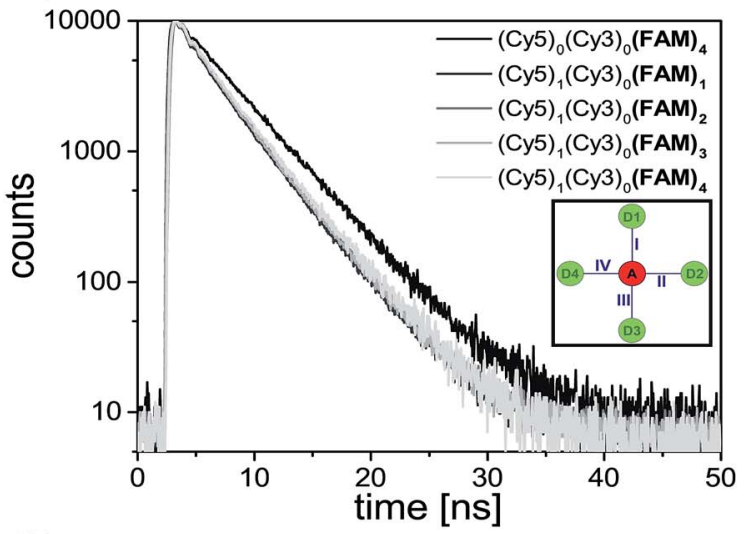

C)

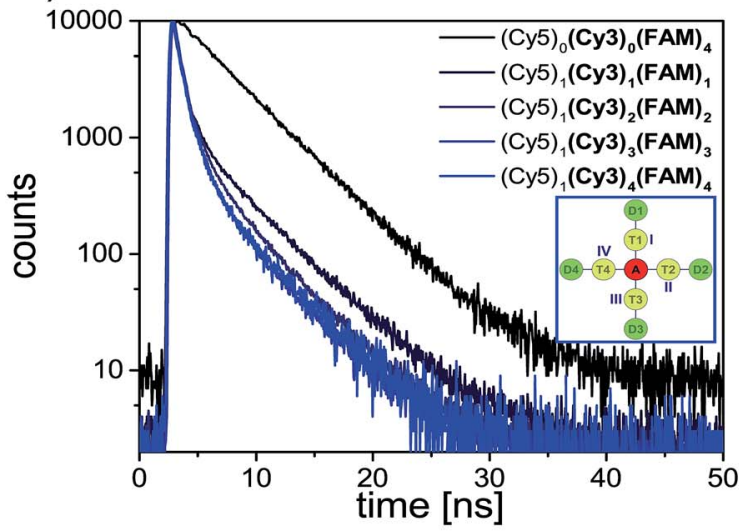

B)

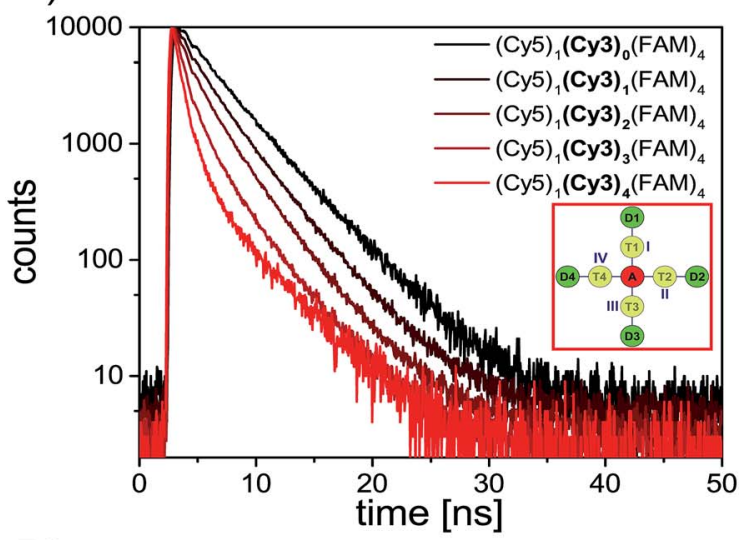

D)

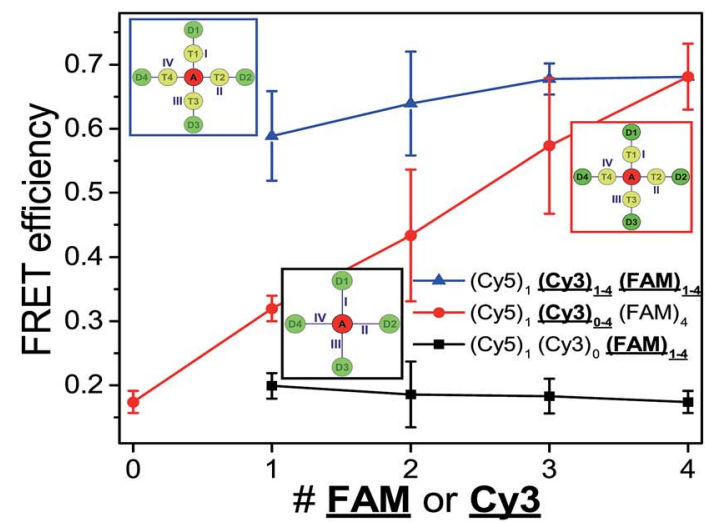

Fig. 3 FAM fluorescence decay curves $\left(\lambda_{\mathrm{ex}}=490 \mathrm{~nm}, \lambda_{\mathrm{em}}=520 \mathrm{~nm}\right.$ ) and FRET efficiencies calculated based on amplitude averaged decay times for different light harvesting systems. (A) FAM fluorescence decay curves for the two-color FRET system with an increasing number of FAM molecules $\left((\mathrm{Cy} 5)_{1}(\mathrm{Cy} 3)_{0}(\mathrm{FAM})_{1-4}\right)$. (B) FAM fluorescence decay curves for the light harvesting system with a fixed number of Cy5 (1) and FAM (4)

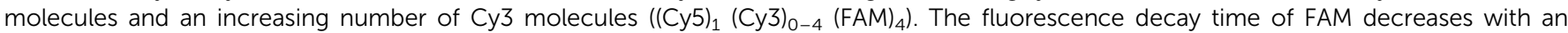
increasing number of Cy3 molecules. (C) FAM fluorescence decay curves for the light harvesting system with an increasing amount of Cy3 and FAM molecules $\left((\mathrm{Cy} 5)_{1}(\mathrm{Cy} 3)_{1-4}(\mathrm{FAM})_{1-4}\right)$. The FAM fluorescence decay time decreases with increasing number of transmitter and donor molecules. (D) FRET efficiencies plotted against the number of FAM and Cy3, respectively (shown in bold letters). For the two-color FRET system, the FRET efficiency decreases slightly with an increasing number of FAM molecules (black). The FRET efficiency increases with an increasing number of FAM and Cy3 for the three-color light harvesting systems $\left((C y 5)_{1}(\mathrm{Cy} 3)_{1-4}(\text { FAM })_{1-4}(\text { blue })_{;}(\text {Cy5 })_{1}(\text { Cy3 })_{0-4}(\text { FAM })_{4}(\right.$ red $)$ ).

The FAM fluorescence decay curves for the light harvesting systems with increasing Cy3 and FAM molecules $\left((\mathrm{Cy} 5)_{1}(\mathrm{Cy} 3)_{1-4}\right.$ $\left.(\mathrm{FAM})_{1-4}\right)$ are depicted in Fig. 3C. Again, the FAM fluorescence decay time decreases with an increasing amount of donor and transmitter molecules. Interestingly, the fluorescence decay time drops drastically from unquenched FAM ((Cy5) $)_{0}(\mathrm{Cy} 3)_{0}$ $\left.(\mathrm{FAM})_{4}: \tau_{\mathrm{D}}=4.3 \mathrm{~ns}\right)$ to the system with just one acceptor, transmitter and donor molecule $\left((\mathrm{Cy} 5)_{1}(\mathrm{Cy} 3)_{1}(\mathrm{FAM})_{1}: \bar{\tau}_{\mathrm{DA}}=\right.$ $(1.8 \pm 0.8) \mathrm{ns})$. This mono-molecular three-color FRET cascade $(\mathrm{Cy} 5)_{1}(\mathrm{Cy} 3)_{1}(\mathrm{FAM})_{1}$ is already very efficient because the spectral overlap of each FRET pair is large (see ESI, Fig S4B and $\mathrm{D}^{\dagger}$ ) and the intermolecular distances are rather short (ca. 3-4 nm). The FAM fluorescence decay time decreases further with an increasing number of transmitter and donor molecules. It has to be noted that the decrease happens to a smaller extent compared to (Cy5) ${ }_{1}(\mathrm{Cy} 3)_{0-4}(\mathrm{FAM})_{4}$ and it reaches a minimum value of $(1.3 \pm 0.2) \mathrm{ns}\left((\mathrm{Cy} 5)_{1}(\mathrm{Cy} 3)_{1}(\mathrm{FAM})_{1}: \bar{\tau}_{\mathrm{DA}}=(1.9 \pm 0.8) \mathrm{ns}\right.$; $(\mathrm{Cy} 5)_{1}(\mathrm{Cy} 3)_{2}(\mathrm{FAM})_{2}: \bar{\tau}_{\mathrm{DA}}=(1.5 \pm 0.4) \mathrm{ns} ;(\mathrm{Cy} 5)_{1}(\mathrm{Cy} 3)_{3}(\mathrm{FAM})_{3}$ :
$\left.\bar{\tau}_{\mathrm{DA}}=(1.4 \pm 0.1) \mathrm{ns} ;(\mathrm{Cy} 5)_{1}(\mathrm{Cy} 3)_{4}(\mathrm{FAM})_{4}: \bar{\tau}_{\mathrm{DA}}=(1.3 \pm 0.2) \mathrm{ns}\right)$. The FRET efficiency is plotted versus the number of $\mathrm{Cy} 3$ and FAM molecules and is shown in Fig. 3D (blue curve). The FRET efficiency rises with an increasing amount of $\mathrm{Cy} 3$ and FAM. The increase in FRET efficiency is not as strong as for $(\mathrm{Cy} 5)_{1}(\mathrm{Cy} 3)_{0-4}$ $(\mathrm{FAM})_{4}$ and is at the beginning $\left((\mathrm{Cy} 5)_{1}(\mathrm{Cy} 3)_{1}(\mathrm{FAM})_{1}\right)$ already quite high. The reason has been explained above. Please note that the position of FAM molecules slightly influences the antenna effect (AE1 and AE2) and FRET efficiency when comparing $(\mathrm{Cy} 5)_{1}(\mathrm{Cy} 3)_{4}(\mathrm{FAM})_{4}(\mathrm{D} 1-\mathrm{D} 4)(\mathrm{AE} 1=0.96 \pm 0.03$, $\mathrm{AE} 2=5.7 \pm 0.4, E=0.68 \pm 0.05)$ and $(\mathrm{Cy} 5)_{1}(\mathrm{Cy} 3)_{4}(\mathrm{FAM})_{4}(\mathrm{D} 5-$ D8) $(\mathrm{AE} 1=0.81 \pm 0.02, \mathrm{AE} 2=4.9 \pm 0.1, E=0.53 \pm 0.07)$ with each other as depicted in Fig. 4.

With these results we have shown that the FRET efficiency is not strongly dependent on the number of transmitter and donor molecules but rather on the spectral properties of the fluorophores (strong increase in FRET efficiency by addition of further transmitter molecules ((Cy5) $\left.\left.{ }_{1}(\mathrm{Cy} 3)_{0-4}(\mathrm{FAM})_{4}\right)\right)$. The 
A)

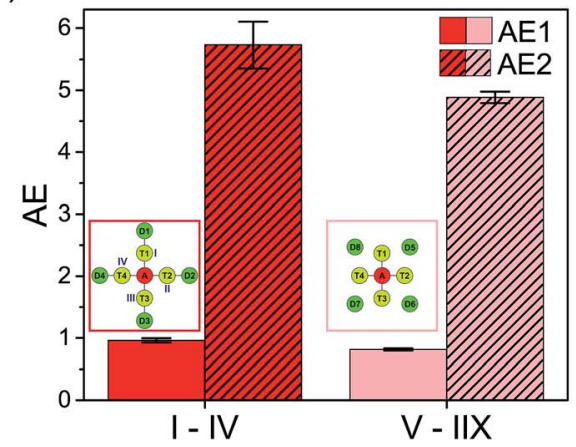

B)

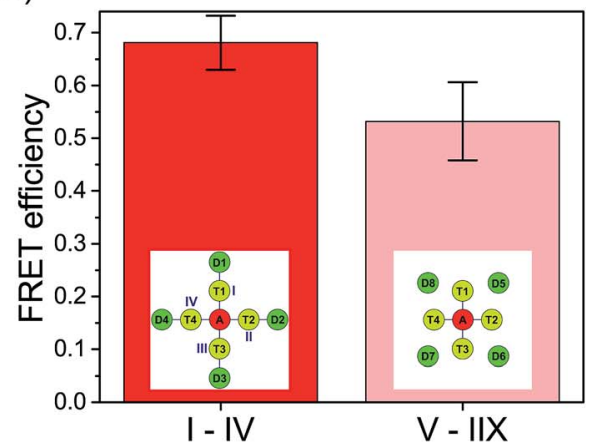

Fig. 4 AE1, AE2 and FRET efficiency for $(\mathrm{Cy} 5)_{1}(\mathrm{Cy} 3)_{4}(\mathrm{FAM})_{4}$ with different donor positions (D1-D4 (red), D5-D8 (rose)). (A) Both AE1 (solid) and AE2 (hatched) are slightly influenced by changing the positions of FAM. (B) The FRET efficiency is also slightly influenced by the position of FAM molecules. The error bars are the standard deviation of three separate measurements.

antenna effect (Fig. 2D) on the other hand highly depends on the number of fluorophores (it rises drastically with an increasing number of transmitter and donor molecules ((Cy5) $\left.\left.(\mathrm{Cy} 3)_{1-4}(\mathrm{FAM})_{1-4}\right)\right)$. In contrast, the FRET efficiency describes the effectivity of donor quenching and the average FRET efficiency can even decrease although the antenna effect is enhanced. This is because the antenna effect only characterizes the enhancement of the acceptor emission, which is achieved by increasing the number of energy-delivering pathways. ${ }^{45}$

\section{Optimization of the light harvesting system}

These design rules can be used to rationally optimize the light harvesting efficiency of artificial antenna systems.

This is demonstrated in the following by further increasing the energy-delivering pathways. Thus, the number of donor molecules is further increased from four to eight. For this, four additional FAM molecules are introduced in such a way that each FAM molecule is placed between two transmitter molecules (see Fig. 1). Here, the distance between FAM and the neighboring Cy3 molecules is approximately the same as for the other positions $(R=3.3$ $\mathrm{nm})$. As shown in Fig. 5A the emission intensity of Cy3 $(565 \mathrm{~nm})$ and Cy5 $(665 \mathrm{~nm})$ increases drastically in presence of eight FAM molecules (gray spectrum) compared to a light harvesting system with only four FAM molecules (red spectrum). The antenna effect (AE1) has been calculated for both systems and is shown in Fig. 5B. With the addition of more FAM molecules the antenna effect 1 is further tuned from $0.96 \pm 0.03$ in $(\mathrm{Cy} 5)_{1}(\mathrm{Cy} 3)_{4}(\mathrm{FAM})_{4}$ to $1.58 \pm 0.08$ in $(\mathrm{Cy} 5)_{1}(\mathrm{Cy} 3)_{4}(\mathrm{FAM})_{8}$. The antenna effect 2 is also higher for $(\mathrm{Cy} 5)_{1}(\mathrm{Cy} 3)_{4}(\mathrm{FAM})_{8}(\mathrm{AE} 2=8.6 \pm 0.2)$ compared to $(\mathrm{Cy} 5)_{1}(\mathrm{Cy} 3)_{4}(\mathrm{FAM})_{4}(\mathrm{AE} 2=5.7 \pm 0.4)$ as shown in Fig. $5 \mathrm{~B}$. This is a remarkable result as it shows that also the second energy transfer step (AE2) is influenced by the presence of dyes emitting further in the blue part of the electromagnetic spectrum. The FAM fluorescence decay curves have been measured for both systems and are depicted in Fig. 5C. The FAM amplitude averaged fluorescence decay time decreases slightly with an increasing number of FAM molecules $\left((\mathrm{Cy} 5)_{1}(\mathrm{Cy} 3)_{4}(\mathrm{FAM})_{4}: \bar{\tau}_{\mathrm{DA}}=(1.3 \pm 0.2) \mathrm{ns}\right.$; $\left.(\mathrm{Cy} 5)_{1}(\mathrm{Cy} 3)_{4}(\mathrm{FAM})_{8}: \bar{\tau}_{\mathrm{DA}}=(1.2 \pm 0.1) \mathrm{ns}\right)$. Hence, the FRET efficiency increases also only slightly with an increasing number of
FAM molecules as shown in Fig. 5D. Again, the antenna effect depends strongly on the number of fluorophores which are present in the light harvesting system. The emission intensity of the final acceptor (Cy5) can be enhanced by adding more and more donor molecules. The FRET efficiency on the other hand is only slightly influenced by further addition of donor molecules. The FRET efficiency as mentioned above describes the degree of donor quenching. Therefore, it is only slightly influenced by the addition of more donor molecules but rather by the addition of further transmitter molecules as shown for $(\mathrm{Cy} 5)_{1}(\mathrm{Cy} 3)_{0-4}(\mathrm{FAM})_{4}$.

To assess the overall light harvesting capability in terms of possible excitation wavelengths, we have measured the excitation spectra of selected light harvesting systems (see Fig. 6). By analyzing excitation spectra, we can determine specific wavelengths at which the light harvesting system is excitable. As shown in Fig. 6A the single acceptor Cy5 $\left((\mathrm{Cy} 5)_{1}(\mathrm{Cy} 3)_{0}(\mathrm{FAM})_{0}\right.$, black spectrum) can only be efficiently excited from $c a$. (570700) $\mathrm{nm}$ (Cy5 excitation peak). By introducing four Cy3 molecules $\left((\mathrm{Cy} 5)_{1}(\mathrm{Cy} 3)_{4}(\mathrm{FAM})_{0}\right.$, Fig. 6A, green spectrum) the system can additionally be excited at wavelengths in the range of the excitation peak of Cy3 $((450-570) \mathrm{nm})$. By extending the light harvesting system even more (introducing FAM) the wavelength range for suitable excitation wavelengths can be further tuned. As shown in Fig. 6A a third excitation peak appears for the mono-molecular three-color FRET cascade $\left((\mathrm{Cy} 5)_{1} \quad(\mathrm{Cy} 3)_{1}\right.$ $(\text { FAM })_{1}$, blue spectrum, FAM: $500 \mathrm{~nm}$, Cy3: $550 \mathrm{~nm}$, Cy5: 650 $\mathrm{nm})$. Thus, the light harvesting complex can be excited at a broader wavelength range. Nevertheless, the overall intensity is rather weak for this dye configuration. This can be further improved by the addition of four FAM molecules ((Cy5) $)_{1}(\mathrm{Cy} 3)_{1}$ $(\mathrm{FAM})_{4}$, Fig. 6A, dark red spectrum). The intensity of the FAM excitation maximum increases due to a higher number of donor molecules. Again, the extent of excitation can be improved by adding more molecules $(\mathrm{Cy} 5)_{1}(\mathrm{Cy} 3)_{4}(\mathrm{FAM})_{4}$ (Fig. 6A, red spectrum). To finally optimize the light harvesting system eight donor molecules are introduced $\left((\mathrm{Cy} 5)_{1}(\mathrm{Cy} 3)_{4}(\mathrm{FAM})_{8}\right.$, Fig. 6A, gray spectrum). With such a light harvesting system the range of possible excitation wavelength is broadened $((425-700) \mathrm{nm})$ and the light energy can be absorbed to a higher extent. To summarize the light harvesting efficiency of the previously 


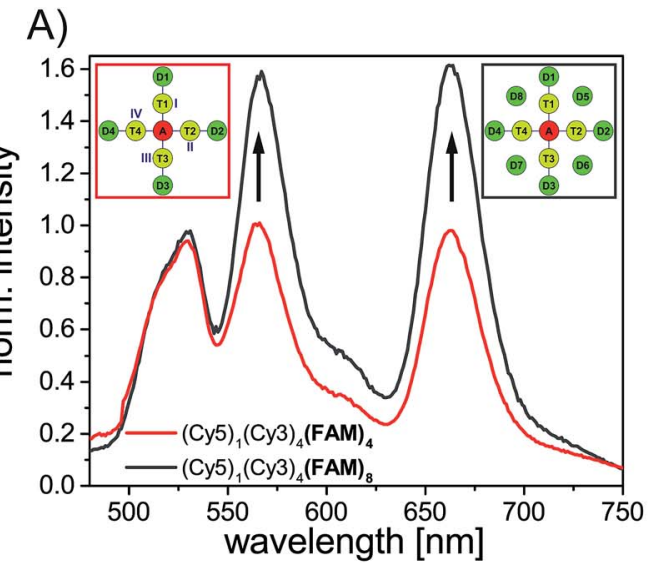

C)

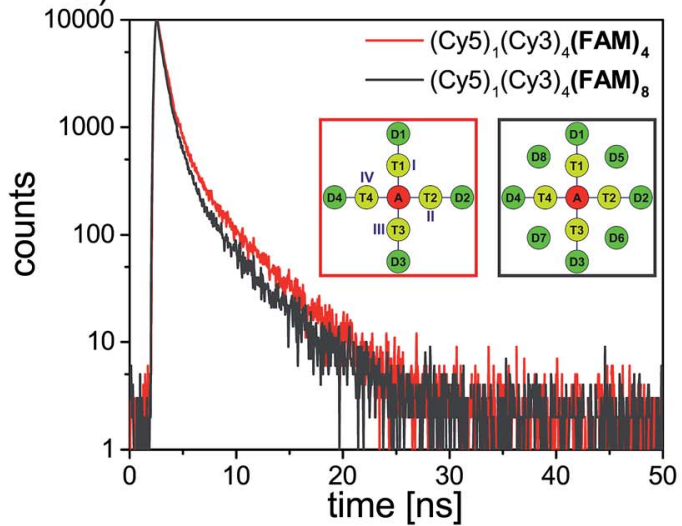

B)

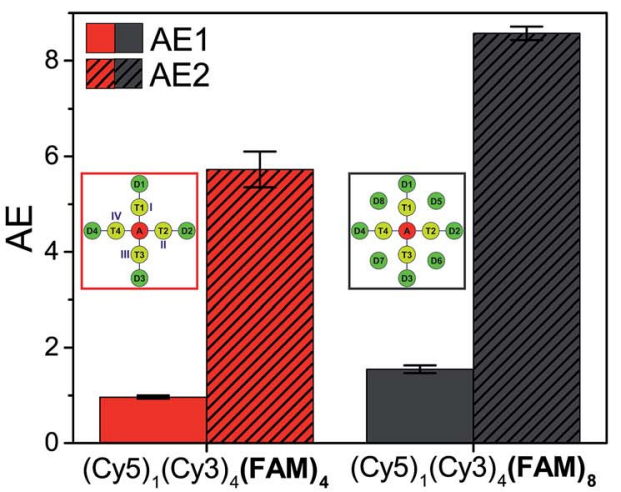

D)

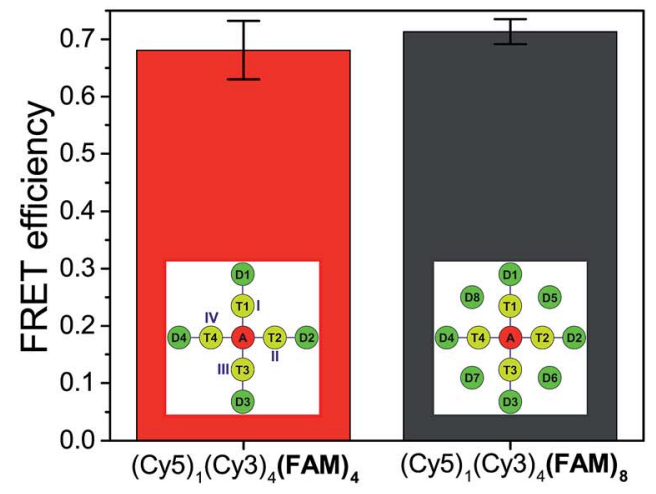

Fig. 5 Steady-state and time-resolved data for $(\text { Cy } 5)_{1}(\text { Cy3 })_{4}(\text { FAM })_{4}($ red $)$ and $(\text { Cy } 5)_{1}(\text { Cy3 })_{4}(\text { FAM })_{8}$ (gray). (A) Normalized emission spectra $\left(\lambda_{\text {ex }}=\right.$ $450 \mathrm{~nm}$ ) for light harvesting systems with four (red) and eight donor molecules (gray). Emission intensities of Cy3 (565 nm) and Cy5 (665 nm) increase with increasing amount of FAM. (B) The antenna effects (AE1 (solid) and AE2 (hatched)) increase with increasing number of FAM molecules. (C) FAM fluorescence decay time $\left(\lambda_{\mathrm{ex}}=490 \mathrm{~nm}, \lambda_{\mathrm{em}}=520 \mathrm{~nm}\right.$ ) decreases slightly with an increasing number of FAM molecules. (D) The FRET efficiency calculated based on the FAM fluorescence decay time is only slightly influenced by an additional number of FAM molecules. The error bars are the standard deviation of three individual measurements.

discussed light harvesting systems, the AE values (AE1 and AE2) are depicted in Fig. 6B. The AE2 value of a two-color FRET-based light harvesting system $\left((\mathrm{Cy} 5)_{1}(\mathrm{Cy} 3)_{4}(\mathrm{FAM})_{0}\right)$, which yields similar values compared to other studies $(\mathrm{AE} 2=2.1 \pm 0.2),{ }^{8} \mathrm{can}$ be significantly improved by the addition of a third blue shifted dye such as FAM $\left(\mathrm{AE} 2(\mathrm{Cy} 5)_{1}(\mathrm{Cy} 3)_{4}(\mathrm{FAM})_{4}=5.7 \pm 0.4\right.$,
A)

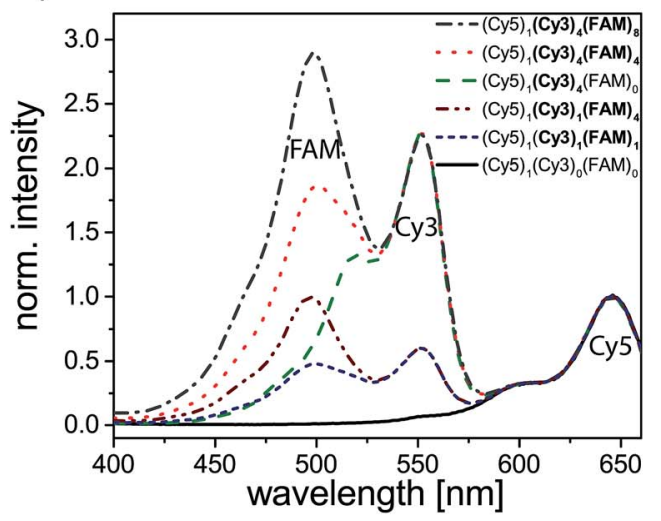

B)

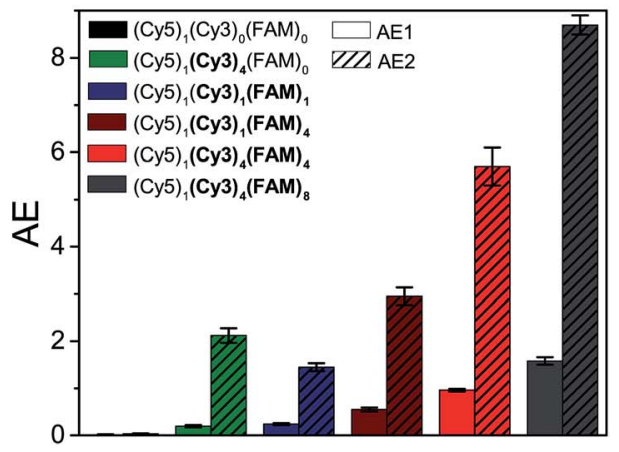

Fig. 6 (A) Excitation spectra $\left(\lambda_{\mathrm{em}}=680 \mathrm{~nm}\right.$ ) for different light harvesting systems showing the impact of additional fluorophores on possible excitation wavelengths. (B) Overview of AE1 and AE2 values for light harvesting systems showing the significant increase due to additional fluorophores. The $A E$ values of $(C y 5)_{1}(C y 3)_{0}(F A M)_{0}$ are rather small $(A E 1=0.02 \pm 0.01, A E 2=0.03 \pm 0.01$ ). The error bars are the standard deviations of different paths/path combinations and three separate measurements, respectively. 
AE2 $(\mathrm{Cy} 5)_{1}(\mathrm{Cy} 3)_{4}(\mathrm{FAM})_{8}=8.7 \pm 0.2$, Fig. 6B). Interestingly, even the system with just one Cy3 molecule and four FAM molecules lead to higher AE2 values compared to $(\mathrm{Cy} 5)_{1}(\mathrm{Cy} 3)_{4}(\mathrm{FAM})_{0}$ in which four Cy3 molecules are present (AE2(Cy5) $)_{1}(\mathrm{Cy} 3)_{4}(\mathrm{FAM})_{0}$ $=2.3 \pm 0.2$ vs. $\mathrm{AE} 2(\mathrm{Cy} 5)_{1}(\mathrm{Cy} 3)_{1}(\mathrm{FAM})_{4}=3.0 \pm 0.2$, Fig. $\left.6 \mathrm{~B}\right)$. In general, Fig. 6B shows that both antenna effects (AE1 and AE2) increase with an increasing number of dye molecules as thoroughly discussed above. This is also in good agreement with the excitation spectra (Fig. 6A) which show an increasing number and intensity of the excitation peaks for an increasing number of fluorophores.

\section{Conclusion}

In conclusion we have created different three-color FRET-based light harvesting systems by changing the amount and ratio of fluorophores (donor: FAM; transmitter: Cy3, acceptor: Cy5). The light harvesting efficiency expressed by the antenna effect and the overall FRET efficiency have been analyzed using steady-state and time-resolved fluorescence spectroscopy. We have shown that the light harvesting efficiency increases for a two color-FRET system by increasing the amount of donor molecules ((Cy5) $)_{1}(\mathrm{Cy} 3)_{0}$ $\left.(\mathrm{FAM})_{0-4}\right)$. The light harvesting efficiency can be further improved by introducing a transmitter dye and changing the transmitter/ donor ratio $\left((\mathrm{Cy} 5)_{1}(\mathrm{Cy} 3)_{0-4}(\mathrm{FAM})_{4}\right)$ or by changing the amount of both transmitter and donor molecules to the same extent $\left((\mathrm{Cy} 5)_{1}(\mathrm{Cy} 3)_{0-4}(\mathrm{FAM})_{0-4}\right)$. Both approaches lead to a linear increase in the antenna effect. The overall FRET efficiency of the two-color FRET system is rather small and is tuned by introducing a transmitter molecule. We have shown that the FRET efficiency rises linearly for $(\mathrm{Cy} 5)_{1}(\mathrm{Cy} 3)_{0-4}(\mathrm{FAM})_{4}$ and only slightly for (Cy5) $(\mathrm{Cy} 3)_{1-4}(\mathrm{FAM})_{1-4}$. We have concluded that the antenna effect is highly dependent on the number of fluorophores involved in the light harvesting system whereas the FRET efficiency depends more on the spectral properties of used fluorophores. The antenna effect describes the efficiency of acceptor sensitization and the FRET efficiency characterizes the degree of donor quenching. Finally, we have shown that we can tune the antenna effect starting from 0.02 in (Cy5) ${ }_{1}(\mathrm{Cy} 3)_{0}(\mathrm{FAM})_{0}$ to 1.58 in (Cy5) $(\mathrm{Cy} 3)_{4}(\mathrm{FAM})_{8}$. Thus, the overall light harvesting efficiency can be enhanced by a factor of almost 80 by changing the stoichiometry of donor and transmitter dyes when excited at $450 \mathrm{~nm}$. For an excitation wavelength of $500 \mathrm{~nm}$ the light harvesting efficiency can be even improved by a factor of 200 . We have shown that by using three different fluorophores in a light harvesting system the range for possible excitation wavelengths is significantly broadened and the light energy can be absorbed to a higher extent. In general, the light harvesting efficiency of a multichromophore system can be optimized based on the following design rules:

(1) To improve the antenna effect, the number of energydelivering pathways must be increased by increasing the number of donor and transmitter molecules. Thus, a rosettelike design is best-suited.

(2) The FRET efficiency can only be increased by optimizing the spectral properties and making the intermolecular distances small. The number of D molecules barely changes the FRET efficiency.
(3) The final energy transfer step is also influenced by the number of dyes which are further blue shifted in the electromagnetic spectrum (addition of an extra donor).

(4) A variety of fluorophores resulting in multiple FRET steps leads to a broader range of possible excitation wavelength.

This can be exploited in artificial light harvesting systems used for instance in photosynthesis, photovoltaics in light harvesting chemical sensors.

\section{Experimental methods}

\section{Materials and chemicals}

Unmodified oligonucleotides (staple strands) have been acquired from Integrated DNA Technologies. The DNA strands have been purified by the manufacturer and dissolved in RNasefree water. The viral genome M13mp18 (7249 nb) has been purchased from tilibit nanosystems $\mathrm{GmbH}$ and dissolved in buffer containing $10 \mathrm{mM}$ Tris and $1 \mathrm{mM}$ EDTA by the manufacturer. The oligonucleotides modified with the organic dyes (FAM, Cy3 and Cy5) have been acquired from Metabion International AG (HPLC purified). All DNA strands have been used as delivered without further treatment. Magnesium chloride $(\geq 98 \%)$ and Tris acetate-EDTA buffer (TAE buffer, 10× concentrated) have been acquired from Sigma Aldrich. The diluted TAE buffer $(1 \times$ concentrated, in ultrapure water (Merck Millipore) $(\mathrm{pH}=8.2)$ contains $40 \mathrm{mM}$ Tris-acetate and $1 \mathrm{mM}$ EDTA. Mica has been purchased from Plano $\mathrm{GmbH}$.

\section{DNA origami preparation}

The triangularly shaped DNA origami structures have been fabricated by mixing the viral genome M13mp18 (5 nM) together with 208 short single DNA strands (150 nM), TAE buffer $(10 \times$ concentrated) containing $100 \mathrm{mM} \mathrm{MgCl}_{2}$ and ultrapure water (Merck Millipore). Using a thermal cycler (PEQLAB/VWR), the solution has been heated up to $80^{\circ} \mathrm{C}$ and then slowly cooled down to $8{ }^{\circ} \mathrm{C}$ in 2 hours with a defined temperature program $\left(80{ }^{\circ} \mathrm{C}-\right.$ $66{ }^{\circ} \mathrm{C}: 1{ }^{\circ} \mathrm{C}$ every $30 \mathrm{~s}, 66^{\circ} \mathrm{C}-25{ }^{\circ} \mathrm{C}: 1{ }^{\circ} \mathrm{C}$ every $2 \mathrm{~min}, 25{ }^{\circ} \mathrm{C}-8{ }^{\circ} \mathrm{C}$ : $1{ }^{\circ} \mathrm{C}$ every minute). Afterwards, the DNA origami structures have been purified using $100 \mathrm{kDa}$ molecular weight cut-off centrifugal filters (Merck Millipore). For this, the samples have been washed four times with TAE buffer $(1 \times$ concentrated) containing $10 \mathrm{mM}$ $\mathrm{MgCl}_{2}$ at $2655 \mathrm{~g}$ for $10 \mathrm{~min}$. Fluorescence spectroscopy measurements have been performed after the DNA origami preparation at a concentration of approximately $5 \mathrm{nM}$.

\section{AFM imaging}

To investigate the correctly formed DNA origami structures, atomic force microscopy (AFM) has been performed for each sample (AFM image is shown in the ESI, Fig S1†). The samples have been adsorbed on freshly cleaved mica (Plano $\mathrm{GmbH}$ ). For this, $2 \mu \mathrm{L}$ of the purified sample ( $c a .20 \mathrm{nM}$ ) and $33 \mu \mathrm{L}$ of TAE buffer $\left(1 \times\right.$ concentrated) containing $10 \mathrm{mM} \mathrm{MgCl}_{2}$ have been incubated for $30 \mathrm{~s}$. After the incubation, the sample has been washed twice with $1 \mathrm{~mL}$ of ultrapure water (Merck Millipore). The fluid has been removed with compressed air. The measurements have been performed in air using the phase contrast mode. AFM 
imaging has been performed with a Flex AFM from Nanosurf GmbH. A cantilever from Budget Sensors (Tap150 Al-G) with a resonance frequency of (125-160) kHz and a spring constant of $5 \mathrm{~N} \mathrm{~m}^{-1}$ has been used to visualize the DNA origami structures.

\section{Steady-state fluorescence spectroscopy}

Steady-state fluorescence spectroscopy measurements have been performed using a FluoromaxP spectrophotometer from HORIBA Jobin Yvon $\mathrm{GmbH}$ with $3 \mathrm{~mm}$ quartz cuvettes from Hellma Analytics. The measurements have been performed in a $90^{\circ}$ angle acquisition using the system internal quantum correction. To record emission spectra, the excitation wavelength has been chosen according to the fluorophore which is directly excited (FAM: $\lambda_{\text {ex }}=450 \mathrm{~nm}$, Cy3: $\lambda_{\mathrm{ex}}=500 \mathrm{~nm}$, Cy5: $\lambda_{\mathrm{ex}}=600 \mathrm{~nm}$ ). To measure excitation spectra, the emission wavelength has been set to $\lambda_{\text {em }}=680 \mathrm{~nm}$. Following settings have been used for all measurements: increment $=1 \mathrm{~nm}$, integration time $=0.2 \mathrm{~s}$, bandpass $($ emission $)=$ bandpass $($ excitation $)=5 \mathrm{~nm}$.

\section{Time-correlated single photon counting}

Time-correlated single photon counting (TCSPC) measurements have been performed on a FLS920 fluorescence spectrophotometer from Edinburgh Instruments Ltd with the F900 software (Edinburgh Instruments Ltd) using $3 \mathrm{~mm}$ quartz cuvettes (Hellma Analytics). The samples have been measured in a $90^{\circ}$ setup. As an excitation source a supercontinuum white light source SC-400-PP from Fianium/NKT Photonics A/S (0.5-20 MHz, $400 \mathrm{~nm}<\lambda<$ $24000 \mathrm{~nm}$, pulse width: ca. $30 \mathrm{ps}$ ) and as a detector a multichannel-plate ELDY EM1-132/300 from Europhoton GmbH have been used. The excitation wavelength has been set to $(490 \pm 1) \mathrm{nm}$ and emission wavelength to $(520 \pm 1) \mathrm{nm}$.

The measured fluorescence decay curves have been fitted using the FAST software (Edinburgh Instruments Ltd). The decay curves have been fitted multi-exponentially (bi- and triexponentially) with the following eqn $\mathrm{S}(1)$.

$$
I(t)=\sum_{i=1}^{n} A_{i} \mathrm{e}^{-\frac{t}{\tau_{i}}}
$$

Here, $\tau_{i}$ is the decay time and $A_{i}$ is the amplitude characteristic for each decay time component. The amplitude averaged decay time $\bar{\tau}_{\mathrm{DA}}$ is then calculated with eqn $\mathrm{S}(2)$.

$$
\bar{\tau}_{\mathrm{DA}}=\frac{\sum_{i=1}^{n} A_{i} \tau_{i}}{\sum_{i=1}^{n} A_{i}}
$$

The last decay time component has been fixed to the unquenched FAM decay time.

\section{Acknowledgements}

This research was supported by the Deutsche Forschungsgemeinschaft (DFG), a Marie Curie FP7 Integration
Grant within the 7th European Union Framework Programme, by the University of Potsdam, the Federal Institute for Materials Research and Testing (BAM), and the DFG project GSC 1013 (SALSA).

\section{References}

1 B. Gobets and R. van Grondelle, Biochim. Biophys. Acta, 2001, 1507, 80-99.

2 R. Emerson, J. Gen. Physiol., 1932, 16, 191-205.

3 G. McDermott, S. M. Prince, A. A. Freer, A. M. Hawthornthwaite-Lawless, M. Z. Papiz, R. J. Cogdell and N. W. Isaacs, Nature, 1995, 374, 517-521.

$4 \mathrm{X}$. Hu, A. Damjanović, T. Ritz and K. Schulten, Proc. Natl. Acad. Sci. U. S. A., 1998, 95, 5935-5941.

5 K. Kalyanasundaram and M. Graetzel, Curr. Opin. Struct. Biol., 2010, 21, 298-310.

6 B. Albinsson, J. K. Hannestad and K. Börjesson, Coord. Chem. Rev., 2012, 256, 2399-2413.

7 F. Garo and R. Häner, Angew. Chem., Int. Ed., 2012, 51, 916-919. 8 E. A. Hemmig, C. Creatore, B. Wunsch, L. Hecker, P. Mair, M. A. Parker, S. Emmott, P. Tinnefeld, U. F. Keyser and A. W. Chin, Nano Lett., 2016, 16, 2369-2374.

9 P. K. Dutta, S. Levenberg, A. Loskutov, D. Jun, R. Saer, J. T. Beatty, S. Lin, Y. Liu, N. W. Woodbury and H. Yan, J. Am. Chem. Soc., 2014, 136, 16618-16625.

10 P. K. Dutta, R. Varghese, J. Nangreave, S. Lin, H. Yan and Y. Liu, J. Am. Chem. Soc., 2011, 133, 11985-11993.

11 A. Ruiz-Carretero, P. G. A. Janssen, A. L. Stevens, M. Surin, L. M. Herz and A. P. H. Schenning, Chem. Commun., 2011, 47, 884-886.

12 A. L. Benvin, Y. Creeger, G. W. Fisher, B. Ballou, A. S. Waggoner and B. A. Armitage, J. Am. Chem. Soc., 2007, 129, 2025-2034.

13 H. Ozhalici-Unal and B. A. Armitage, ACS Nano, 2009, 3, 425433.

14 T. Mayr, S. M. Borisov, T. Abel, B. Enko, K. Waich, G. Mistlberger and I. Klimant, Anal. Chem., 2009, 81, 65416545.

15 L. Olejko, P. J. Cywinski and I. Bald, Nanoscale, 2016, 8, 10339-10347.

16 S. Buckhout-White, C. M. Spillmann, W. R. Algar, A. Khachatrian, J. S. Melinger, E. R. Goldman, M. G. Ancona and I. L. Medintz, Nat. Commun., 2014, 5, 5615.

17 M. Heilemann, P. Tinnefeld, G. Sanchez Mosteiro, M. Garcia Parajo, N. F. van Hulst and M. Sauer, J. Am. Chem. Soc., 2004, 126, 6514-6515.

18 I. H. Stein, C. Steinhauer and P. Tinnefeld, J. Am. Chem. Soc., 2011, 133, 4193-4195.

19 C. M. Spillmann, S. Buckhout-White, E. Oh, E. R. Goldman, M. G. Ancona and I. L. Medintz, Chem. Commun., 2014, 50, 7246-7249.

20 P. W. K. Rothemund, Nature, 2006, 440, 297-302.

21 F. Zhang, J. Nangreave, Y. Liu and H. Yan, J. Am. Chem. Soc., 2014, 136(32), 11198-11211.

22 I. Bald and A. Keller, Molecules, 2014, 19, 13803-13823. 
23 J. Prinz, B. Schreiber, L. Olejko, J. Oertel, J. Rackwitz, A. Keller and I. Bald, J. Phys. Chem. Lett., 2013, 4, 4140-4145. 24 J. Prinz, C. Heck, L. Ellerik, V. Merk and I. Bald, Nanoscale, 2016, 8, 5612-5620.

25 J. Prinz, A. Matkovic, J. Pesic, R. Gajic and I. Bald, Small, 2016, 12, 5458-5467.

26 A. Keller, J. Rackwitz, E. Cauët, J. Liévin, T. Körzdörfer, A. Rotaru, K. V. Gothelf, F. Besenbacher and I. Bald, Sci. Rep., 2014, 4, 7391.

27 J. Rackwitz, J. Kopyra, I. Dąbkowska, K. Ebel, M. L. Ranković, A. R. Milosavljević and I. Bald, Angew. Chem., Int. Ed., 2016, 55, 10248-10252.

28 S. Vogel, J. Rackwitz, R. Schürmann, J. Prinz, A. R. Milosavljevic, M. Refregiers, A. Giuliani and I. Bald, J. Phys. Chem. Lett., 2015, 6, 4589-4593.

29 Z. Deng, A. Samanta, J. Nangreave, H. Yan and Y. Liu, J. Am. Chem. Soc., 2012, 134, 17424-17427.

30 I. H. Stein, V. Schüller, P. Böhm, P. Tinnefeld and T. Liedl, ChemPhysChem, 2011, 12, 689-695.

31 L. Olejko, P. J. Cywinski and I. Bald, Angew. Chem., Int. Ed., 2015, 54, 673-677.

32 Y. Ke, T. Meyer, W. M. Shih and G. Bellot, Nat. Commun., 2016, 7, 10935.

33 R. Tsukanov, T. E. Tomov, Y. Berger, M. Liber and E. Nir, J. Phys. Chem. B, 2013, 117, 16105-16109.
34 B. Saccà, Y. Ishitsuka, R. Meyer, A. Sprengel, E.-C. Schöneweiß, G. U. Nienhaus and C. M. Niemeyer, Angew. Chem., Int. Ed., 2015, 54, 3592-3597.

35 B. L. Cannon, D. L. Kellis, P. H. Davis, J. Lee, W. Kuang, W. L. Hughes, E. Graugnard, B. Yurke and W. B. Knowlton, ACS Photonics, 2015, 2, 398-404.

36 R. M. Zadegan, M. D. E. Jepsen, L. L. Hildebrandt, V. Birkedal and J. Kjems, Small, 2015, 11, 1811-1817.

37 A. Adronov, S. L. Gilat, J. M. J. Fréchet, K. Ohta, F. V. R. Neuwahl and G. R. Fleming, J. Am. Chem. Soc., 2000, 122, 1175-1185.

38 R. A. Miller, A. D. Presley and M. B. Francis, J. Am. Chem. Soc., 2007, 129, 3104-3109.

39 F. Pu, L. Wu, X. Ran, J. Ren and X. Qu, Angew. Chem., Int. Ed., 2015, 54, 892-896.

40 S. L. Gilat, A. Adronov and J. M. Fréchet, Angew. Chem., Int. Ed., 1999, 38, 1422-1427.

41 L. Stryer and R. P. Haugland, Proc. Natl. Acad. Sci. U. S. A., 1967, 58, 719-726.

42 D. W. Brousmiche, J. M. Serin, J. M. J. Fréchet, G. S. He, T.-C. Lin, S.-J. Chung, P. N. Prasad, R. Kannan and L.-S. Tan, J. Phys. Chem. B, 2004, 108, 8592-8600.

43 T. Förster, Naturwissenschaften, 1946, 33, 166-175.

44 T. Förster, Ann. Phys., 1948, 437, 55-75.

45 B. Jana, S. Bhattacharyya and A. Patra, Nanoscale, 2016, 8, 16034-16043. 